Revue d'histoire de l'Amérique française

REVUE D.HISTOIRE DE L'AMÉRIQUE FRANÇAISE

\title{
La république des hommes : les Patriotes de 1837 face aux femmes
}

\section{Allan Greer}

Volume 44, numéro 4, printemps 1991

URI : https://id.erudit.org/iderudit/304922ar

DOI : https://doi.org/10.7202/304922ar

Aller au sommaire du numéro

\section{Éditeur(s)}

Institut d'histoire de l'Amérique française

\section{ISSN}

0035-2357 (imprimé)

1492-1383 (numérique)

Découvrir la revue

Citer cet article

Greer, A. (1991). La république des hommes : les Patriotes de 1837 face aux femmes. Revue d'histoire de l'Amérique française, 44(4), 507-528.

https://doi.org/10.7202/304922ar
Résumé de l'article

Cet article examine la dimension sexuelle de la Rébellion de 1837 dans le Bas-Canada et soutient que les femmes furent bien moins actives dans la lutte anti-gouvernementale qu'elles ne l'avaient été au XVIII ${ }^{\mathrm{e}}$ siècle dans diverses émeutes et autres mouvements de résistance. L'explication de ce changement est liée au procès de " masculinisation » de la politique qui a lieu un peu partout en Occident à la même époque. Comme le républicain type de la première moitié du XIX ${ }^{\mathrm{e}}$ siècle, le patriote du Bas-Canada défend une conception essentiellement masculine de la citoyenneté en régime démocratique. D'autre part la conviction rousseauiste que la femme vertueuse doit se confiner dans son rôle domestique permet de comprendre les poussées occasionnelles de mysoginie contre les femmes qui affichent publiquement leur opposition au mouvement patriote. 


\title{
LA RÉPUBLIQUE DES HOMMES: LES PATRIOTES DE 1837 FACE AUX FEMMES ${ }^{1}$
}

\author{
ALLAN GREER \\ Département d' histoire \\ Université de la Colombie Britannique
}

\section{RÉSUMÉ}

Cet article examine la dimension sexuelle de la Rébellion de 1837 dans le Bas-Canada et soutient que les femmes furent bien moins actives dans la lutte anti-gouvernementale qu'elles ne l'avaient été au XVIII ${ }^{e}$ siècle dans diverses émeutes et autres mouvements de résistance. L'explication de ce changement est liée au procès de «masculinisation» de la politique qui a lieu un peu partout en Occident à la même époque. Comme le républicain type de la première moitié du XIX ${ }^{e}$ siècle, le patriote du Bas-Canada défend une conception essentiellement masculine de la citoyenneté en régime démocratique. D'autre part la conviction rousseauiste que la femme vertueuse doit se confiner dans son rôle domestique permet de comprendre les poussées occasionnelles de mysoginie contre les femmes qui affichent publiquement leur opposition au mouvement patriote.

\section{ABSTRACT}

This article explores the gender dimension of the Lower Canadian Rebellion of 1837. Women, it is argued, were much less active in the anti-government cause than they had been in various 18th century episodes of riot and resistance. The explanation for this change focuses on a process of masculinization of politics to be found at work in most parts of the western world in this period. As typical republicans of the first half of the nineteenth century, the Lower Canadian patriots championed a specifically male conception of democratic citizenship. At the same time, their Rousseauian conviction that virtuous women should confine themselves to domestic matters, helps explain their occasional misogynist outbursts against women who took public stands against the patriot movement.

1 Je remercie Mariana Valverde, Bruce Curtis, Ed Hundert et Micheline Dumont d'avoir bien voulu commenter une première version de cet article. Je remercie également le Conseil de recherches en sciences humaines du Canada de son appui ainsi que mon assistant de recherche, Irshad Manji. 
«Je vois que cette funeste philosophie gâtes [sic] toutes les têtes et le contrat social de Rousseau te fait oublier l'Évangile de StPaul. "Femmes soyez soumises à vos maris».»"

(Louis-Joseph Papineau à sa femme, 1830)

"Voulez-vous donc connoître les hommes, étudiez les femmes. Cette maxime est générale, et jusque-là tout le monde sera d'accord avec moi. Mais si j'ajoute qu'il n'y a point de bonnes moeurs pour les femmes hors d' une vie retirée et domestique; si je dis que les paisibles soins de la famille et du ménage sont leur partage, que la dignité de leur sexe est dans sa modestie, que la honte et la pudeur sont en elles inséparables de l'honnêteté, que rechercher les regards des hommes c'est déjà s'en laisser corrompre, et que toute femme qui se montre se déshonore; à l'instant va s'élever contre moi cette philosophie d'un jour, qui naît et meurt dans le coin d'une grande ville, et veut étouffer de là le cri de la nature et la voix unanime du genre humain.» ${ }^{3}$

(Jean-Jacques Rousseau)

La reine Victoria monte sur le trône d'Angleterre en août 1837, au moment où s'accroissent les tensions qui vont bientôt provoquer une insurrection armée dans le Bas-Canada. Rien n'indique que la jeune souveraine de dix-sept ans s'intéresse beaucoup aux querelles politiques qui déchirent ses colonies d'Amérique du Nord, mais les célébrations en l'honneur de son couronnement donnent aux Canadiens l'occasion de manifester leurs vifs sentiments pour ou contre leur lien avec la couronne britannique. C'est le Te Deum ordonné à la mi-août par l'évêque de Montréal, comme le veut la coutume dans de semblables occasions, qui soulève le plus de controverses. À Saint-Polycarpe, où le curé ose prononcer quelques mots de louange à l'endroit de la nouvelle reine, les patriotes locaux réussissent à interrompre la cérémonie.

Le Te Deum avait à peine commencé que le peuple quittait l'église en corps, ne laissant que les femmes et les marguilliers en compagnie de monsieur le curé. L'assistant du bedeau commençait à sonner les cloches à ce moment-là, mais les paroissiens l'en ont empêché en lui disant que les cloches étaient leur propriété, non celle de la reine d'Angleterre, et qu'elles ne devaient pas sonner... ${ }^{4}$

2 Cité dans Micheline Dumont et al., L'histoire des femmes au Québec depuis quatre siècles (Montréal, Les Quinze, 1982), 149. Il est vrai que cette remarque est dite sur le ton de la plaisanterie, mais, comme nous le verrons, elle traduit bien les convictions profondes de Papineau.

3 «Lettre à $\mathrm{M}$. d'Alembert sur son article Genève dans le VII ${ }^{e}$ volume de l'Encyclopédie, et particulièrement sur le projet d'établir un thêâtre de comédie en cette ville», Euvres complètes de J.-J. Rousseau (Paris, Alexandre Houssiaux, 1853), III: 151.

$4 \quad$ The Vindicator, $1^{\text {er }}$ septembre 1837 , traduction. 
À noter le vocabulaire utilisé par The Vindicator pour décrire l'incident, l'opposition entre «le peuple» et «les femmes». La moitié de la population de la province pourrait lire de mauvais présages dans ces mots, qui émanent des presses d'un journal dédié au principe de la souveraineté du peuple!

Il ne s'agit pas là d'une maladresse journalistique comme le prouvent bien les connotations sexuelles des protestations qui accueillent le Te Deum du couronnement. Dans la paroisse de Contrecœur, un marchand radical sort de l'église à la tête des autres paroissiens en criant: «C'est bien de la peine de chanter le Te Deum pour la sacrée Reine, sacré putain qui a les jambes en l'aire.» On rapporte qu'un patriote de Nicolet a harangué la population sur le parvis de l'église en ces termes: «Que quant au roi, ce n'était qu'un zéro, à qui les canadiens payaient une pension... Preuve que les rois ne sont que des zéros on était actuellement gouvernés par une jeune reine de dix sept ans.» Plus tard, au moment des combats de novembre et de décembre, un sympatisant américain des patriotes s'attire des ennuis à SaintAthanase pour avoir «ridiculisé la personne de Sa Majesté en déclarant que les loyalistes étaient gouvernés par une fillette, qu'ils étaient gouvernés par un jupon» ${ }^{5}$. Un tel langage ne peut manquer de choquer les radicaux anglais, bien disposés envers la jeune reine qui leur semble un personnage plus sympathique que son célèbre oncle Guillaume $\mathrm{IV}^{6}$. Parallèlement, ces déclarations montrent l'importance qu'il faut accorder à la dimension sexuelle pour bien comprendre le mouvement patriote et la rébellion de 1837-1838.

Il n'est guère étonnant de voir les patriotes s'opposer à la monarchie, à la domination britannique dans le Bas-Canada et, par conséquent, à la reine. Mais pourquoi s'en prennent-ils, de façon aussi blessante et personnelle, au sexe de Victoria? Si l'on considère tous les milieux républicains de l'époque, il est certain que les patriotes ne sont pas les seuls à se montrer sensibles aux distinctions de sexe. Des recherches récentes montrent que la différence entre les hommes et les femmes était une préoccupation majeure des écrivains politiques et des révolutionnaires de la fin du XVIII ${ }^{e}$ siècle et du début du XIX ${ }^{\mathrm{e}}$.

5 Archives nationales du Québec, collection événements de 1837-1838 (ci-après ANQ, 1837), $\mathrm{n}^{\circ}$ 324, déposition du Baron Augustin de Diemar, 21 décembre 1837; ibid., no 242, déposition de Joseph-Louis Pinard, $1^{\text {er }}$ février 1838, ibid., déposition de Thomas Casson, 31 décembre 1838 (cf, ibid., n 1483, Lacombe à Walcott, 23 août 1837). De leur côté les loyalistes ont adopté une attitude chevaleresque, jurant de «faire tous les sacrifices nécessaires au maintien de l'autorité légitime de notre jeune et belle reine». Discours de l'honorable McGill, Montréal, 23 octobre 1837, Jean-Paul Bernard, dir., Assemblées publiques, résolutions et déclarations de 1837-1838 (Montréal, VLB éditeur, 1988), 239.

6 Dorothy Thompson, communication personnelle. 
Dans la mesure où ils s'opposent aux hiérarchies existantes pour des motifs égalitaires et revendiquent le gouvernement du «peuple», les philosophes, les Jacobins et les patriotes d'Amérique doivent s'attaquer à la question de savoir qui forme «le peuple». Il n'est sûrement pas constitué de tous les êtres humains qui habitent un territoire donné, c'est-à-dire que tous ne partagent pas également de la même façon l'autorité souveraine. On tend à exclure notamment les femmes de toute participation directe à la vie politique de la cité républicaine. Les déclarations peuvent être criptiques et ouvertes à de multiples interprétations, remplies de sous-entendus et de silences, mais il n'en reste pas moins qu'à l'époque des grandes révolutions bourgeoises, le sexe devient de plus en plus la première ligne de démarcation entre les gouvernants et les gouvernés. Ceci survient en partie par défaut, au fur et à mesure que les anciens clivages politiques basés sur la naissance ou autres statuts privilégiés sont remis en cause. Mais la position découle également d'une conception républicaine de la citoyenneté, qui est essentiellement masculine.

Inspirés par une certaine interprétation de l'histoire de la Grèce et de la Rome antiques, des républicains modernes comme Jean-Jacques Rousseau croient que seuls les hommes sont qualifiés pour assumer les responsabilités du citoyen ${ }^{7}$. Ils sont plus qualifiés pour la guerre et, croit-on, tous les bons citoyens doivent être prêts à défendre leur pays sur le champ de bataille. Rousseau est surtout convaincu que les hommes sont par nature plus aptes à subordonner leur égoïsme et leurs intérêts particuliers au bien de la collectivité. À l'opposé, les femmes sont nécessairement associées à la procréation et aux soins des enfants; par conséquent, elles sont vouées à la famille et elles ne peuvent renoncer à cette allégeance innée sans agir contre leur nature. Moins gênés par les liens affectifs, les hommes peuvent acquérir cette vertu civique, c'est-à-dire le dévouement envers le bien commun, indispensable à toute saine république. Il importe de noter, cependant, que Rousseau ne considère pas les femmes comme inférieures aux hommes. Il accorde au contraire une grande valeur à la sphère domestique remplie d'amour et de soins attentifs, où se déploie la vraie vocation des femmes. Son enthousiasme pour la maternité et le bonheur conjugal souligne le fait qu'à ses yeux, le rôle familial des femmes constitue le complément indispensable du rôle actif de citoyen joué par les hommes. La vie domestique et la vie publique, laisse-t-il

7 Plusieurs études l'ont démontré, mais j'ai trouvé particulièrement utile celle de Joel Schwartz, The Sexual Politics of Jean-Jacques Rousseau (Chicago, University of Chicago Press, 1984). Les principaux textes sont: Discours sur l'origine et le fondement de l'inégalité parmi les hommes, Du contrat social, «Lettre à M. d'Alembert» et Émile. 
entendre, sont deux éléments d'égale importance de toute vie civilisée. Pour remplir son devoir, la femme républicaine doit faire preuve d'une vertu particulière; ce qui la caractérise, ce n'est pas son ardeur pour la chose publique, mais «son innocence et sa pudeur» et, pour garantir aux yeux du monde sa conduite monogame, elle doit se confiner dans la vie privée ${ }^{8}$.

Rousseau ne peut être qualifié simplement de chauviniste mâle; en fait, ses défenseurs contemporains insistent pour dire qu'il reconnaît aux femmes un pouvoir légitime énorme, même s'il s'agit d'un pouvoir caché qu'elles exercent grâce à leur influence sexuelle sur certains hommes ${ }^{9}$. En outre, parce que Rousseau insiste sur la liberté et sur le développement de la personnalité, il est possible de voir en lui le père intellectuel de la libération de la femme moderne. Il suffit d'étendre aux femmes le raisonnement que le philosophe applique à «l'homme» et des conséquences troublantes s'ensuivent. (La citation en tête de cet article prouve bien qu'au moins un citoyen du BasCanada perçoit de telles possibilités chez Rousseau!) Il n'en demeure pas moins que Rousseau lui-même ne présente pas une interprétation féministe des idées de Jean-Jacques. Dans tous ses écrits, ce sont les besoins des hommes qui prédominent et les femmes n'apparaissent sous un jour favorable que dans la mesure où elles les secondent.

Si je me suis attardé sur Rousseau, ce n'est pas parce que ses écrits constituent un code de conduite pour les patriotes du BasCanada, mais parce qu'il est l'un des rares écrivains de son époque à accorder une attention constante et explicite à la dimension sexuelle de la politique. Sans chercher à nier l'originalité de son génie, il semble juste de croire que plusieurs éléments essentiels de sa pensée au sujet des femmes se retrouvent dans le mouvement républicain international. On peut détecter dans les journaux des patriotes certains échos de sa thèse voulant que les hommes et les femmes possèdent des traits moraux complémentaires, mais fondamentalement différents. Ce point ressort très clairement d'un article de La Minerve publié sous le titre «Les deux républiques»:

Le monde moral est tout mixte, composé d'hommes et de femmes, et il ne doit qu'à ce mélange la plupart de ses coutumes, de ses usages et de ses cérémonies; s'il n'y avait plus de femmes dans l'espace humaine [sic], les hommes ne seraient plus recon-

8 Joan B. Landes, Women and the Public Sphere in the Age of the French Revolution (Ithaca, Cornell University Press, 1988).

9 Joel Schwartz, The Sexual Politics... 
naissables. Ce n'est qu'en s'efforçant de plaire à l'autre sexe qu'ils viennent à bout de se polir... Les femmes, de leur côté, rapportent tout à cette autre moitié du genre humain, à laquelle elles se trouvent unies... c'est au désir de lui plaire qu'elles doivent cet air gracieux, ces yeux qui disent tant de choses, cette rougeur modeste qui embellit leur teint; cette voix si douce et touchante. Enfin ce désir réciproque est donc dans les deux sexes un instinct précieux qui tend à les perfectionner l'un et l'autre; aussi voit-on qu'un homme, qui compte les femmes pour rien, devient ordinairement un sauvage: de même qu'une femme destinée par la nature à s'entendre et à figurer avec l'homme ne peut guère [le] haïr ni le mépriser sans devenir une créature farouche et insupportable. ${ }^{10}$

De tels sentiments sont loin de se manifester uniquement dans les cercles républicains. En fait, l'idée fondamentale selon laquelle les femmes doivent se consacrer à la vie domestique (valorisée) tandis que les hommes doivent gouverner l'État et la communauté, prévaut partout où la bourgeoisie parvient à prédominer au cours des XVIII et $\mathrm{XIX}^{\mathrm{e}}$ siècles. Au début de la Révolution française, par exemple, on encourage un peu la participation politique des femmes, mais cette tendance est rapidement suivie d'une politique d'exclusion absolue de la vie publique, sous prétexte que leurs incursions dans le royaume mâle de la politique active nuisent autant à la vie politique qu'à la famille. Cette attitude des Jacobins conduit Olympe de Gouges, féministe et royaliste, à protester en ces termes: «Les femmes sont aujourd'hui respectées et exclues; sous l'Ancien Régime, elles étaient méprisées et puisssantes» ${ }^{11}$. Antérieurement, les femmes américaines avaient été traitées de la même façon pendant «leur» guerre révolutionnaire ${ }^{12}$.

Pourtant, dans l'ordre nouveau, les femmes ne sont pas toujours «respectées», comme le prouvent les propos misogynes et licencieux des patriotes contre la reine Victoria. A cet égard également, le comportement des Canadiens du Bas-Canada semble refléter l'attitude

$10 \quad$ La Minerve, 27 juillet 1836.

11 Darline Gay Levy, Harriet Branson Applewhite et Mary Durham Johnson, eds., Women in Revolutionary Paris, 1789-1795 (Urbana, University of Illinois Press, 1979); Sîan Reynolds, «Marianne's Citizens? Women, the Republic and Universal Suffrage in France», Women, State and Revolution: Essays on Power and Gender in Europe Since 1789 (Amherst, University of Massachusetts Press, 1987), 101-122; Joan B. Landes, Women and the Public Sphere.... La citation d'Olympe de Gouges est de Dorinda Outram, «Le langage mâle de la vertu: Women and the Discourse of the French Revolution», Peter Burke et Roy Porter, eds., The Social History of Language (Cambridge, Cambridge University Press, 1987), 126.

12 Linda Kerber, Women of the Republic: Intellect and Ideology in Revolutionary America (Chapel Hill, University of North Carolina Press, 1980). 
générale de l'époque, l'attitude républicaine en particulier. Dans une perspective que l'on pourrait qualifier de «rousseauisme vulgaire», la femme qui se confine dans son rôle domestique est vénérée, mais celle qui s'aventure dans le monde politique suscite une profonde méfiance. Non seulement la nature n'a-t-elle pas doté les femmes des dons requis pour s'occuper des affaires publiques, mais leur tentative d'y participer met directement en danger la vie sacrée du couple. Cette attitude s'explique par le fait que, dans le discours républicain, la vie publique va littéralement de pair avec une performance publique sous les regards de tous. Si cette visibilité garantit la vertu des hommes, c'est le contraire dans le cas des femmes. S'afficher en public répugne à une femme honnête parce que c'est là une preuve d'immoralité au même titre que sa réclusion dans l'espace privé est la preuve de sa pudeur. Comme l'écrit Rousseau «l'audace chez la femme est le signe certain de sa déchéance». Les deux significations de l'expression «femme publique» - une personne politiquement importante et une prostituée - finissent par se confondre dans la pensée républicaine de l'époque ${ }^{13}$.

L'inconduite sexuelle des femmes, signalée par une participation active à la politique, est évidemment jugée déplorable pour toutes sortes de raisons, mais il importe de voir que, du point de vue républicain, elle représente des dangers politiques précis. Lorsque les femmes s'éloignent du foyer, elles ne peuvent pas soutenir leurs époux ou élever leurs fils pour qu'ils deviennent de bons citoyens. Et surtout, elles agissent contre leur nature chaste et modeste. Compte tenu de leur importance et de leur grande ascendance sur les hommes, leur inconduite a pour résultat de dénaturer les hommes, de les rendre efféminés et, par conséquent, vulnérables à la tyrannie. C'est ainsi que les républicains tendent à associer la corruption politique dans le monde masculin, soit la principale menace contre la liberté, à la corruption des mœurs chez les femmes ${ }^{14}$. C'est en reconnaissant l'exis-

13 Joan B. Landes, Women and the Public Sphere.... La citation de Rousseau est à la page 75 de cet ouvrage.

14 Dorina Outram, «Le langage mâle». On peut trouver une magnifique illustration de cette perspective dans l'œuvre d'un contemporain britannique de Rousseau, républicain comme lui, Edward Gibbon. Dans le chapitre 20 de The Decline and Fall of the Roman Empire, Gibbon consacre plusieurs longues pages corsées à l'inconduite de l'impératrice Théodora au sixième siècle. Il explique que Justinien, son mari, commit par amour la grande erreur de l'associer au pouvoir au lieu d'en faire uniquement son épouse. Longtemps avant de réussir à séduire Justinien, poursuit-il, Théodora était renommée à Constantinople et bien au-delà pour la beauté de ses traits et de son corps. «But this form was degraded by the facility with which it was exposed to the public eye, and prostituted to licentious desire. Her venal charms were abandoned to a promiscuous crowd of citizens and strangers, of every rank and of every profession: the fortunate lover who had been promised a night of enjoyment was often driven from her bed by a stronger or more wealthy favorite...» Etc. etc. 
tence de ce lien entre le rôle public de la femme, le désordre sexuel, la corruption politique et la tyrannie, que nous commençons à comprendre pourquoi il peut venir à l'esprit des patriotes d'accuser la reine Victoria (innocente et jeune mais indubitablement un personnage public important) d'être une «putain».

Parvenu à ce point, un lecteur pourrait objecter que ni Rousseau ni les patriotes n'ont inventé le patriarcat. Sans aucun doute, l'idée que «la place de la femme est à la maison» sous l'autorité de son mari est fort ancienne, comme nous le rappelle Papineau lorsqu'il cite les Écritures pour réprimer «l'indépendance» de sa femme. Ce qui est nouveau au début du XIX ${ }^{e}$ siècle, c'est l'insistance mise sur «le culte du rôle domestique des femmes» et, parallèlement, le monopole des affaires publiques que s'attribuent les hommes. Dans la tradition antérieure (que l'on pourrait appeler dans un sens général «l'ancien régime»), la situation n'était pas aussi tranchée, surtout dans le domaine politique, et rien ne justifiait le principe selon lequel les hommes devaient monopoliser le pouvoir souverain de l'État uniquement parce qu'ils étaient des hommes. La plupart des gens étaient considérés comme des sujets et, sous cet angle, hommes et femmes étaient politiquement sur un pied d'égalité. Par contre, certaines femmes - de madame de Pompadour en France à madame Péan au Canada - exerçaient une grande influence sur les affaires de l'État ${ }^{15}$.

Selon les idéologues de l'absolutisme, la masse n'était pas censée avoir voix au chapitre en politique, mais elle pouvait toujours faire valoir son point de vue par des manifestations, des émeutes ou des insurrections. De plus, les femmes jouaient souvent un rôle important dans ces événements. Dans le Canada français du XVIII ${ }^{\mathbf{e}}$ siècle, les femmes ont souvent été à l'avant-garde dans plusieurs confrontations entre les habitants et les autorités religieuses au sujet des limites paroissiales et, pendant la guerre de Sept ans, ce sont les femmes surtout, qui protestèrent contre la pénurie et le rationnement ${ }^{16}$. Plus tard, lorsque l'insurrection éclata dans les campagnes du Bas-Canada pendant l'occupation américaine, en 1775, les femmes ont joué encore

15 À propos de la Nouvelle-France et des aventures de Madeleine de Verchères, seigneuresse et hérö̈ne militaire, Colin Coates écrit: «This social system, though patriarchal, allowed certain women to wield a great deal of power.» Il aurait pu ajouter "ouvertement» afin de mieux distinguer le $\mathrm{XVIII}^{e}$ siècle du XIX' ${ }^{e}$ siècle, alors que les hommes s'attendaient encore à ce que les femmes dans l'élite exercent un pouvoir, mais secrètement, en privé. Colin Coates, «Authority and Illegitimacy in New France: the Burial of Bishop Saint-Vallier and Madeleine de Verchères vs. the Priest of Batiscan», Histoire sociale/Social History, 22 (mai 1989): 65-90.

16 Louise Dechêne, Habitants et marchands de Montréal au XVII siècle (Paris et Montréal, Plon, 1974), 464; Terrence Crowley, “"Thunder Gusts": Popular Disturbances in Early French Canada», Société historique du Canada, Communications historiques (1979): 19-22. 
une fois un rôle important à plusieurs endroits. À Pointe-auxTrembles, deux femmes sont allées de porte en porte noircir le visage de tous leurs voisins qui avaient collaboré aux levées de milice par les autorités britanniques. Ailleurs, on a signalé le fait suivant:

La veuve Gabourie surnômée la reine d'Hongrie a fait plus de mal dans cette paroisse qu'aucun autre; elle tenait souvant chez elle des assemblées où elle présidait, tandant à soulever les esprits contre le gouvernement et à les animer en faveur des rebels. Pour mieux parvenir à son but détestable elle leur faisait boire des liqueurs fortes. ${ }^{17}$

Pendant des années, les femmes du Bas-Canada ont bénéficié du droit de vote, du moins dans une certaine mesure ${ }^{18}$. Dans la loi de 1791 qui créait le gouvernement colonial, le droit de vote aux élections de l'Assemblée législative était accordé en fonction des biens fonciers, sans mention du sexe des propriétaires. Par conséquent, des femmes qui possédaient des propriétés (généralement des veuves) ont souvent voté. Cependant, le suffrage féminin n'était pas reconnu par tous. Les «poll books» qui ont été conservés montrent que, selon la date et le comté en cause, il pouvait y avoir un nombre important d'électrices ou absolument aucune ${ }^{19}$. Nul doute que certains agents d'élection interdisaient aux femmes de voter «à cause de leur sexe». Dans un cas, ce geste a soulevé des protestations de la part des hommes dont le candidat pouvait tirer profit du suffrage féminin. «C'est la propriété et non la personne, soutiennent-ils, qui est le fondement de la représentation dans le gouvernement anglais», un argument en faveur du vote des femmes qui ne peut être qualifié, dans les circons-

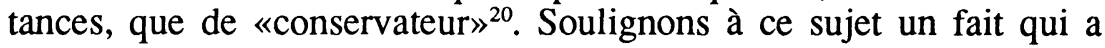

17 «Journal... pour l'examen des personnes qui ont assisté ou aidé les rebels... 1776», Rapport de l'Archiviste de la province de Québec (1927-28): 480. Voir aussi, ibid., 447, 450, 470 et 496.

18 W. R. Riddell, "Woman Franchise in Quebec, a Century Ago», Société royale du Canada, Proceedings and Transactions, 22, section 2 (1928): 85-99; Fernand Ouellet, Le BasCanada, 1791-1840: changements structuraux et crise (Ottawa, Éditions de l'Université d'Ottawa, 1976), 42-43; David De Brou, «Mass Political Behaviour in Upper-Town Quebec, 1792-1836», thèse de Ph.D., Université d'Ottawa, 1989, 94-98.

19 En plus des travaux cités précédemment, voir Archives nationales du Canada (ANC), Registres des élections du Bas-Canada, vol. 21; dans le comté de Québec, 1804 voix (aucune femme); dans le comté de Charlesbourg, 1817 voix (aucune femme); dans le village de William Henry, 1827 voix (trois femmes ont demandé de voter et une demande a été refusée). Par contre, à l'élection de 1832 dans le quartier ouest de Montréal, Fernand Ouellet a trouvé 199 femmes parmi les 1533 noms.

20 Pétition de divers électeurs, Haute-Ville de Québec, 1828, A. G. Doughty et N. Story, eds., Documents Relating to the Constitutional History of Canada, 1819-1828 (Ottawa, King's Printer, 1935), 520. 
échappé à l'attention des historiens nationalistes féministes: cette acceptation partielle et contestée des voix des femmes n'a pas existé uniquement dans le Bas-Canada. Au XVIII ${ }^{e}$ siècle, en GrandeBretagne et dans ses colonies, les femmes ont pu voter à certaines occasions et, en France, elles ont participé à l'élection des représentants aux États généraux de 1789.

Pour les députés du Bas-Canada, le spectacle des femmes participant aux élections semble de plus en plus anormal et, en 1834, ils adoptent une loi qui leur retire officiellement ce droit. À noter que cette mesure ne soulève aucune controverse et ne semble même pas très importante aux yeux de ceux qui l'appuient. En fait, l'article de la loi sur les élections qui supprime le vote des femmes reçoit moins d'attention que d'autres articles traitant de l'assermentation et de la nomination des agents d'élection. Il est proposé au départ par John Neilson, un député modéré de Québec qui s'est dissocié de Papineau et des patriotes les plus radicaux, et Papineau use de toute son influence pour le faire adopter en dépit de sa mésentente profonde avec Neilson. Nulle part, je n'ai pu retracer la moindre objection à l'exclusion des femmes du processus électoral. Dans les journaux du Bas-Canada de toutes tendances (inutile de dire qu'ils sont rédigés par des hommes, à l'intention des hommes), cette décision est passée sous silence ou bien traitée comme une simple mesure d'ordre administratif ${ }^{21}$. Ainsi, même dans ces années 1830 , turbulentes et violemment partisanes, il y a des questions sur lesquelles tous les partis peuvent s'entendre!

En fait, Louis-Joseph Papineau est le seul membre de l'Assemblée qui prend la peine de justifier cette exclusion, dont la nécessité semble aller de soi pour ses collègues. En présentant son argumentation contre le vote des femmes, il importe de noter qu'il n'invoque aucune incapacité de jugement ou de compréhension politique, qui justifierait leur disqualification. Ce qui inquiète Papineau, c'est plutôt le danger, que représente l'exercice public du suffrage des femmes, pour l'ordre sexuel dans la sphère domestique. (Rappelons que l'adoption du vote secret est encore loin dans l'avenir). «Il est ridicule, déclare-t-il, il est

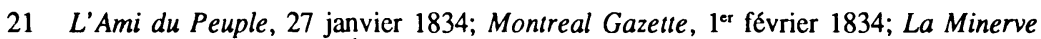
3 février 1834. Le Canadien et $L^{\prime} E ́ c h o ~ d u$ Pays ne font aucune mention du retrait du droit de vote féminin. Notons qu'après avoir été adopté par l'Assemblée législative, le projet de loi de 1834 sur les élections est bloqué par la suite à différentes étapes du processus législatif pour des raisons qui n'ont rien à voir avec la clause sur le vote des femmes. Le projet semble n'avoir jamais eu force de loi. Néanmoins, les femmes ne semblent plus monter à la tribune pour voter, après 1834. Ne voulant prendre aucune chance, le parlement du Canada-Uni adopte en 1849 une loi qui interdit aux femmes de voter. 
odieux de voir trainer aux hustings des femmes par leur mari, des filles par leur père, souvent même contre leur volonté. L'intérêt public, la décence, la modestie du sexe exigent que ces scandales ne se répètent plus.»22 $\mathrm{Ne}$ nous attardons pas sur ce qu'il y a d'ironique à voir cet homme se poser en galant protecteur de la fragilité des femmes au moment où il réduit considérablement leurs droits politiques. Ce qui me semble impossible de passer sous silence dans ce discours de Papineau, et ce qui est caractéristique des républicains, c'est la façon dont il associe trois éléments: 1 - «l'intérêt public»; 2 - la pudeur et «la modestie» des femmes; 3 - le retrait des femmes de l'arène politique. Une telle association est évidemment conforme aux idées de Rousseau. Si les hommes politiques de toute allégeance sont fondamentalement d'accord sur le rapport des femmes à la politique, il semble dans l'ordre des choses que ce soit un radical qui prenne la position la plus ferme.

Il y a pourtant des contre-courants, une possibilité, bien mince il est vrai, d'élaborer différemment le discours républicain sur les femmes et sur les droits politiques. Dans le Bas-Canada, comme en Europe et aux États-Unis, on peut utiliser à des fins proto-féministes le discours sur l'égalité, et même celui sur les «sphères séparées». À la veille de la rébellion, une rare abonnée de La Minerve fait appel au nationalisme des patriotes pour qu'ils reconnaissent l'égalité des sexes comme une caractéristique propre à la nation canadienne-française ${ }^{23}$. Adélaïde (il est courant d'utiliser un pseudonyme dans les lettres adressées au rédacteur) commence par souligner une pratique anglaise qui s'insinue sournoisement dans l'ancien cérémonial canadien qui entoure la signature du contrat de mariage. Lorsqu'on fait appel à elles comme témoins, il semble que certaines femmes mariées de Montréal signent de leur prénom suivi du nom de leur mari. L'abandon par ces femmes de l'usage de signer de leur nom de jeune fille perturbe Adélaïde car cela laisse entrevoir des changements profonds dans la conception du mariage. L'utilisation du nom de famille porté avant le mariage est liée à un système légal et moral qui permet à une femme mariée de conserver ses biens et son identité. Adélaïde souligne la nette différence à cet égard entre le Bas-Canada et l'Angleterre où, croit-elle, les épouses ne peuvent posséder de biens et sont entièrement sous la dépendance de leurs maris. Chez les Canadiens français, la gestion de la propriété familiale et l'éducation des enfants se font en «collaboration mutuelle» (cette expression est soulignée dans le

22 La Minerve, 3 février 1834. Voir aussi, Montreal Gazette, ler février 1834.

23 Ibid., 2 février 1837. 
texte original). «Nos lois, d'accord avec nos mœurs, font de la femme l'associée de l'homme aussi bien que sa compagne.»

Les idées d'Adélaïde sur le droit conjugal et la coutume ne représentent pas une description fidèle de la réalité. Il faut plutôt les considérer comme un programme, un énoncé normatif qui insiste sur les tendances des Canadiens français en faveur de l'égalité des sexes, tout en passant fort à propos sous silence leurs inclinations contraires. Adélaïde omet de mentionner, par exemple, que si l'époux et l'épouse jouissent à parts égales du bien familial selon la coutume de Paris, la loi dit clairement par ailleurs que «l'homme est le maître de la communauté» ${ }^{24}$. Il est vrai que le droit civil canadien protège les droits de propriété de la veuve et des enfants héritiers des deux sexes beaucoup mieux que le droit anglais. Ainsi, le pater familias canadien-français ne dispose pas de la marge de manœuvre économique et des menaces de déshéritement pour intimider ses «dépendants», au même titre que ces tyrans domestiques qui foisonnent dans les romans britanniques du XVIII ${ }^{e}$ siècle. Toutefois, cela ne signifie pas que le Canada français ne soit pas une société patriarcale, mais simplement que le pouvoir mâle se fonde sur d'autres instruments de domination. Ainsi (et ce n'est là qu'un des éléments du patriarcat dont les ramifications sont trop nombreuses pour que nous les analysions ici), sous le Régime français, toute désobéissance des épouses et des enfants pouvait, en principe, faire l'objet de poursuites au criminel $^{25}$.

Il n'en reste pas moins que l'institution de la communauté de biens et les règles successorales de la Coutume de Paris qui imposent le partage égal entre héritiers des deux sexes, constituent d'importantes sauvegardes pour les filles et les épouses, même si dans d'autres domaines, ces dernières sont soumises à la domination masculine. Pourtant, au cours des décennies qui ont précédé la rébellion, on a assisté à l'érosion de ces sauvegardes. La plupart des contemporains n'ont sans doute pas remarqué ces changements, qui sont apparus très graduellement, mais de plus en plus d'habitants s'arrangent pour léguer la totalité ou la plus grande partie de leur propriété à leurs fils, laissant la sécurité matérielle de leurs filles aux soins de leurs futurs époux ${ }^{26}$. Cet abandon d'une partie des règles du droit civil canadien-

24 François-Joseph Cugnet, An Abstract of those Parts of the Custom of the Viscounty and Provostship of Paris, Which Were Received and Practised in the Province of Quebec, in the Time of the French Government (London, 1772), 56; Allan Greer, Peasant, Lord and Merchant: Rural Society of Three Quebec Parishes, 1740-1840 (Toronto, University of Toronto Press, 1985), 53-56.

25 John F. Bosher, «The Family in New France», Barry Gough, ed., In Search of the Visible Past (Waterloo, Wilfrid Laurier University Press), 9.

26 Allan Greer, Peasant, Lord and Merchant..., 80-81, 223. 
français s'explique par un certain nombre de facteurs, notamment par la pénurie croissante de terres offertes à la colonisation. L'influence de la tradition juridique anglaise peut aussi avoir joué un rôle en renforçant l'idée qu'un homme doit être en mesure de disposer de "son» bien comme il l'entend (selon la proposition tacite, mais fondamentale, voulant que le bien familial soit sous l'autorité du père/mari). Cela explique peut-être pourquoi la personne qui signe Adélaïde s'inquiète tellement de l'adoption d'usages anglais dans un domaine mineur en comparaison, celui de la signature des contrats de mariage.

Quoi qu'il en soit, il est clair qu'Adélaïde présente un véritable plaidoyer en faveur d'une plus grande égalité des sexes. Elle le fait dans les seuls termes susceptibles de plaire aux lecteurs de $L a$ Minerve, ceux du républicanisme nationaliste. Comme d'autres féministes pionnières, notamment Wollstonecraft, elle ne peut pas remettre en question l'idée du rôle domestique de la femme qui prévaut alors. Elle insiste plutôt sur les avantages de l'harmonie, de la collaboration mutuelle et de l'égalité dans la sphère privée de la vie conjugale. De toute évidence, sa lettre n'est pas une argumentation en faveur de «l'indépendance» féminine au sens où l'on entendra ce mot à la fin du $\mathrm{XX}^{e}$ siècle, mais dans le monde réel où vit Adélaïde, bien des choses devront changer pour que tous les couples puissent vivre ensemble dans la coopération et le respect mutuel véritables.

Son appel aux hommes du parti des patriotes est-il entendu? Non, si l'on en juge par l'absence de réactions à sa lettre dans les numéros subséquents de La Minerve. Le comportement des radicaux pendant la rébellion n'illustre pas non plus leur profond attachement à la conception que se fait Adélaïde de l'égalité conjugale des Canadiens français. Quand la question de la communauté de biens est soulevée en 1837-1838, c'est que certains patriotes exigent son abolition, sous prétexte qu'elle constitue une violation du droit de l'homme à administrer librement «son» bien ${ }^{27}$ ! De surcroît, on relève dans les documents relatifs à l'insurrection au moins un cas d'épouse violemment battue par un rebelle notoire. À la fin de 1838, Jean-Baptiste Lague de Saint-Mathias bat sans pitié, à plus d'une occasion, sa femme enceinte, Marie-Desanges Brunette. L'accusant d'être une «bureaucrate», il menace de la tuer ou de la faire arrêter par les patriotes. Mal lui en prend puisque Marie, aidée par une voisine, se rend auprès des autorités pour leur révéler les activités révolutionnaires clandestines de Lague ${ }^{28}$. Dans un cas de ce genre, il est difficile de

27 Voir La Minerve, 14 août 1837.

28 ANQ, 1837, $\mathrm{n}^{\circ} 1666$, déposition de Marie Desanges Brunette, 2 décembre 1838. Cf., ibid., $\mathrm{n}^{\circ}$ 1665, déposition d'Agathe Vient, 30 décembre 1838. 
démêler les raisons personnelles et politiques qui incitent Madame Lague à dénoncer son mari; en fait, sa prise de position politique (si elle est vraiment une constitutionnaliste aussi convaincue que le prétend Jean-Baptiste) et son attitude à l'égard de son conjoint sont sans aucun doute inextricablement mêlées. La violence de Lague est peutêtre exceptionnelle (comment savoir s'il était courant de battre sa femme à l'époque?), mais son désaccord politique avec sa femme ne pourrait-il pas indiquer une tendance générale chez les hommes et les femmes du Canada français, au temps de la rébellion, à diverger d'opinion politique?

Cela dit, il importe de noter que la crise de l'été de 1837 débute par une tentative assez gauche de la part des patriotes d'obtenir la collaboration active des femmes. C'est à l'occasion de la campagne de boycott des importations britanniques que les radicaux demandent à leurs femmes et à leurs mères de les appuyer. Ce boycott et la mobilisation subséquente des femmes s'inspirent de l'expérience de l'Amérique pré-révolutionnaire, ce que Papineau et ses associés n'ignorent pas $^{29}$. Ils considèrent que les femmes ont un rôle clé à jouer, en premier lieu à titre de consommatrices. Comme les tissus représentent une bonne part des exportations britanniques au Canada, la presse patriote juge bon de sermonner les dames du pays pour qu'elles renoncent à leurs parures étrangères en faveur des simples étoffes de fabrication domestique.

\begin{abstract}
Women of Canada! Little as ye think it, it is in your power partly to avert the awful storm now threatening us with such terrific fury... There is a demon rapidly destroying your country's prosperity, the virtue of your children and the happiness of your own hearts. You feel it not; you know it not; yet is it a thousand times more terrible in its influence than the all-devouring serpent in days of yore... The demon that I speak of, that hath taken possession of, and sullied, the purity of your minds, is an inordinate love of dress...
\end{abstract}

Sans nous attarder sur la question de savoir comment, avec un style pareil, on peut prétendre défendre la cause de la simplicité et de la modestie, il faut cependant souligner l'association typiquement républicaine faite entre le salut national et la modestie féminine. L'article se termine par un appel vibrant aux femmes du Bas-Canada: «Throng not your city's streets clothed in foreign apparel. Let the leading ones among you assume the dress of humble toile, and, believe me, by so

29 Voir le discours de Papineau tel qu'il est cité dans The Vindicator, 6 juin 1837. Voir aussi, Linda Kerber, Women of the Republic... 
doing, they will have tended more to the preservation of their country's rights and trade, than the numberless resolutions of the grey-beards and councillors. $\rangle^{30}$

Qui est censé filer et tisser cette toile patriotique? Sûrement pas les vieilles barbes et les conseillers! La campagne contre les importations exige avant tout la mobilisation des femmes dans le rôle de productrices domestiques. Dans certaines parties de la province, notamment dans la vallée du Richelieu, les femmes relèvent ce défi. À Saint-Charles, dit-on, «les femmes même partageaient l'enthousiasme... et elles se mirent à se rivaliser d'industrie pour faire les plus beaux tissus. $\rangle^{31}$ Ignorées auparavant dans le discours des patriotes, les femmes commencent à figurer souvent dans la presse radicale, mais habituellement de façon anonyme et stéréotypée. Ainsi, au cours d'un banquet à Contrecœur, cinquante-six hommes portent un toast à «Josephte, la femme de Jean-Baptiste, aussi patriote que belle, aussi agréable que vertueuse; elle contribuera puissament au bonheur du pays par son industrie et ses efforts pour encourager les manufactures domestiques ${ }^{32}$.

On commence par enlever à Josephte son droit de vote, puis on lui demande de peiner à son métier à tisser pour libérer le pays! Il serait tentant de traiter avec cynisme l'appel que les patriotes lancent aux femmes. Mais, la position de ces radicaux est plus complexe et à mesure que la crise de 1837 s'amplifie, elle se préoccupe davantage du statut des citoyennes. Un journal reproduit des extraits de l'étude de Harriet Martineau sur l'inexistence politique des femmes ${ }^{33}$. Il signale aussi en termes favorables la formation d'associations patriotiques féminines dans plusieurs paroisses rurales. On sait peu de choses sur ces associations, mais en septembre 1837, elles semblent élargir leurs intérêts pour englober d'autres questions en plus du boycott.

\section{HONOR TO OUR PATRIOTIC LADIES}

A party two hundred and fifty ladies, belonging to the parish of St. Antoine, celebrated, on Thursday, the 10th inst. their love of

30 The Vindicator, 27 juin 1837. Mentionné ici dans le contexte spécifique du boycott des produits britanniques, le penchant des femmes pour les vêtements somptueux est un des thèmes favoris des chroniqueurs bourgeois du XIX ${ }^{e}$ siècle. Voir Mariana Valverde, «The Love of Finery: Fashion and the Fallen Woman in Nineteenth-Century Social Discourse», Victorian Studies, 32 (hiver 1989): 169-188.

31 ANC, MG24, B82, "Quelques notes historiques sur les événements politiques de 1837 en Canada», 9.

$32 \quad$ La Minerve, 17 août 1837.

33 The Vindicator, 4 juillet 1837. 
country and their patriotism, by a public dinner, from which every imported article was rigidly excluded. Various patriotic toasts, appropriate to the spirit of the times, were proposed. A guard of honor, composed of forty men belonging to the parish, was in attendance with their muskets, and occasionally complimented the fair party by firing a feu de joie. So far from being frightened by the smell of powder, or the roar of musketry, many of the ladies, to prove that they can act in case of need, took the muskets and discharged them with the most admirable tact and courage. When the women of Canada are displaying such patriotism, it is not very probable the men will be backward when the day of trial comes. ${ }^{34}$

Personne ne s'attend évidemment à ce que les femmes prennent les armes dans la lutte à venir. Néanmoins, au-delà du traitement condescendant accordé aux «dames patriotiques», on perçoit une reconnaissance de l'importance de la contribution féminine à la mobilisation nationale. Comme d'autres radicaux de la classe moyenne dans d'autres pays, les patriotes se voient forcés, par la situation révolutionnaire elle-même, de modifier quelque peu leurs vues afin d'attirer les femmes dans le mouvement. Des raisons analogues expliquent leur attitude plus démocratique à l'égard des pauvres. Toutefois, en ce qui concerne les femmes, ils ressentent peut-être plus profondément l'ambivalence d'une telle ouverture, parce que les relations en cause sont plus intimes que les relations de classes. Par conséquent, tout en tentant de stimuler et de favoriser la participation politique des femmes, la presse radicale laisse percer, par ses références incessantes à leur faiblesse et à leur beauté, son inquiétude au sujet des dangers que pose le renversement de la hiérarchie des sexes. Non sans appréhension, les patriotes mâles élargissent le concept maléable du rôle domestique féminin de façon à permettre aux femmes de collaborer directement à la lutte nationale; par contre, ils ne renoncent absolument pas à l'idéologie des sphères distinctes.

Un fait indéniable demeure: le mouvement des patriotes est fondamentalement masculin, tant par son style que par son orientation philosophique. Lors des assemblées de protestation de l'été 1837, les orateurs insistent sur les thèmes de l'indépendance, de l'honneur et des vertus masculines et leurs appels s'adressent clairement aux hommes. Il est certain que des femmes assistent à ces assemblées; en fait, les journaux constitutionnalistes se plaisent à signaler qu'elles réunissent surtout des femmes et des enfants. La réaction de la presse patriote est révélatrice. Au lieu de réfuter l'idée que la présence des

34 Ibid., 22 septembre 1837. 
femmes pourrait enlever tout sérieux à ces assemblées, The Vindicator préfere minimiser leur participation: «les belles dames patriotiques» n'auraient fait qu'agiter des mouchoirs par les fenêtres des maisons environnantes ${ }^{35}$.

Ce genre de déclarations n'est guère propre à renforcer les tentatives des patriotes pour rallier les femmes, pas plus sans doute que l'anticléricalisme notoire de leur mouvement. A cette époque, dans le Bas-Canada comme dans d'autres parties du monde catholique, la religion est en voie de devenir la responsabilité particulière des femmes au sein de la famille ${ }^{36}$. Des données sur la composition des diverses confréries de dévotion confirment cette tendance. Minoritaires dans l'ensemble de ces associations au XVII ${ }^{e}$ siècle, les femmes deviennent nettement prédominantes dans la première moitié du XVIII e et cette tendance va en s'accentuant par la suite ${ }^{37}$. Même si la hiérarchie de l'Église officielle demeure strictement masculine, les femmes de toutes les catégories sociales tendent à nouer des relations privilégiées avec le clergé. Malgré son autoritarisme, l'Église fait une place aux femmes, place qu'elles ne trouvent pas dans le mouvement républicain.

Il n'est guère étonnant que, dans l'ensemble, les femmes n'embrassent pas la cause des patriotes avec beaucoup d'enthousiasme. Une telle affirmation ne vise aucunement à nier ou à atténuer les souffrances qu'endureront des centaines de femmes lorsque leurs biens seront pillés, leurs maisons réduites en cendres et leurs maris emprisonnés à la suite des batailles de 1837 et de 1838 . Nous savons aussi qu'en plus de leur participation à la campagne contre les importations, les femmes brodent des bannières, cachent des fugitifs et aident les insurgés de bien d'autres façons; une paroissienne de SaintBenoît devient même célèbre pour les chansons satiriques qu'elle compose contre son curé, un «chouayen» loyaliste ${ }^{38}$. Pourtant, mise à part ces rôles complémentaires, les preuves d'une participation active des femmes à la cause des patriotes sont presque inexistantes. Aucun des 1356 noms qui figurent sur la liste officielle des prisonniers poli-

35 Voir, par exemple, le récit des assemblées de Sainte-Scholastique et de Napierville dans The Vindicator, 6 juin et 25 juillet 1837.

36 Jean-Pierre Wallot, Un Québec qui bougeait: trame socio-politique au tournant du $X I X^{*}$ siècle (Sillery, Boréal, 1973), 203.

37 Marie-Aimée Cliche, Les pratiques de dévotion en Nouvelle-France: comportements populaires et encadrement ecclésial dans le gouvernement de Québec (Québec, Presses de l'Université Laval, 1988), 181-183, 208, 232.

38 Marcelle Reeves-Morache, "La Canadienne pendant les troubles de 1837-1838», Revue d'histoire de l'Amérique française, 5,1 (juin 1951): 99-117; Micheline Dumont et al., L'histoire des femmes au Québec..., 143-149. 
tiques de la période semble appartenir à une femme ${ }^{39}$. La même impression ressort des milliers de dépositions et des autres sources narratives. Nulle part les femmes ne jouent un rôle marquant ou même très actif, que ce soit à titre de combattantes, d'espionnes, d'agitatrices ou de journalistes ${ }^{40}$. Même s'il est vrai que les femmes sont généralement ignorées dans les sources que privilégient les historiens, ce serait une grave erreur que d'attribuer ce silence généralisé seulement au caractère biaisé de la documentation.

Des femmes sont présentes dans ces sources, mais elles le sont surtout parce qu'elles s'opposent aux patriotes et à leur insurrection. Dans les témoignages de douzaines de témoins et de prisonniers, il est fait mention de femmes qui ont supplié leur mari de rester à la maison plutôt que de joindre le camp des rebelles. Les contemporains imputent évidemment cette attitude à la nature même du sexe faible, mais on peut se demander si ces femmes n'auraient pas fait preuve de plus d'audace si la cause les avait inspirées davantage. Il n'existe sûrement aucune raison de penser que les femmes du Canada français n'appuient pas pleinement la rébellion à cause d'une timidité naturelle ou de leur «conservatisme». Ce que nous savons de leurs activités antigouvernementales au XVIII ${ }^{e}$ siècle suffit pour rejeter cette hypothèse. De toute évidence, quelque chose a changé entre 1775 , lorsque la «reine d'Hongrie» se met à la tête des paysans de l'île d'Orléans, et 1837 , lorsque les forces antigouvernementales ne peuvent afficher aucune héroïne. Un nouveau facteur est évidemment le mouvement des patriotes qui, en dépit de ses efforts mitigés pour encourager les associations féminines, défend nettement un univers politique masculin, où les femmes ne sont pas vraiment les bienvenues. Nul doute également que bien des femmes ne souhaitent pas le moindrement participer aux luttes politiques pour ou contre le régime colonial britannique du Bas-Canada. Après tout, l'idéologie des deux sphères séparées n'a pas cours seulement chez les patriotes et elle n'est pas

39 Irish University Press Series, British Parliamentary Papers, Colonies, Canada, 14: 405-425. Évidemment, l'homme risquait davantage d'être arrêté que la femme, même si tous deux commettaient le même délit politique. Mais, en dépit du biais inhérent à ces chiffres sur les arrestations, il est quand même étonnant qu'aucune femme ne figure parmi les prisonniers politiques.

40 Certains ont tenté (voir les travaux de Reeves-Morache, de Dumont et collaboratrices, cités précédemment) de faire d'Émilie Boileau de Chambly la Madeleine de Verchères du XIX ${ }^{c}$ siècle, mais leurs arguments ne sont pas du tout convaincants. Ils semblent reposer sur un passage des mémoires de R. S. M. Bouchette dans lequel l'auteur décrit une réunion des patriotes dans la maison d'Émilie et de son mari, Timothée Kimber, pendant la crise de 1837. Bouchette a été impressionné en voyant Madame Kimber tenir un pistolet. Toutefois, rien n'indique qu'elle ait jamais utilisé ou brandi cette arme au cours de confrontations avec les forces antipatriotes; en fait, il n'y a aucune raison de croire qu'elle ait même jamais sorti cette arme de chez elle. 
non plus l'apanage exclusif des hommes. À mesure qu'elle gagne du terrain, des deux côtés de l'Atlantique ${ }^{41}$, les femmes elles-mêmes tendent de plus en plus à souscrire à l'idée que les affaires de la nation doivent être laissées aux hommes.

Cependant, toutes les femmes ne sont pas disposées à accepter le rôle qui leur est assigné. En fait, pendant la période de tension qui précède la rébellion, deux hérö̈nes modelées sur l'image de Madeleine de Verchères deviennent célèbres dans toute la province parce qu'elles n'hésitent pas à s'engager dans de dangereuses confrontations armées. L'une est Hortense Globensky, la femme de Guillaume Prévost, notaire de Sainte-Scholastique. C'est une partisane convaincue du parti constitutionnel, qui s'attire la haine de tous les patriotes locaux au cours des agitations de juillet dans le comté des DeuxMontagnes. Saisissant un fusil à l'approche d'une foule hostile qui, croit-elle, vient piller sa maison, elle organise la défense de son foyer de façon si efficace que la foule renonce à poursuivre l'attaque et se retire. Même à l'automne de 1837 , elle ne craint pas de faire irruption dans les assemblées patriotes pour dénoncer la sédition. Son habitude de brandir, à ces occasions, un pistolet chargé lui attire des ennuis; les patriotes de Sainte-Scholastique la dénoncent aux autorités pour possession illégale d'arme à feu. De leur côté, les constitutionnalistes de Montréal portent Madame Prévost aux nues parce qu'elle a prouvé que «même une femme» peut mettre une foule poltronne en fuite. Ils expriment leur admiration en lui offrant une théière en argent sur laquelle est gravée l'inscription suivante: «...in memory of her heroism, greater than that expected of a woman... ${ }^{42}$

Une championne encore plus redoutable du parti constitutionnel est Rosalie Cherrier, dite «Madame St-Jacques» de Saint-Denis ${ }^{43}$. Comme Hortense Globensky, elle s'oppose courageusement et ouvertement aux radicaux au cœur même du fief patriote, mais, pour elle, il n'y aura pas de théière en argent; sa vie personnelle va si outrageusement à l'encontre des conventions qu'il est hors de question que la respectable presse conservatrice lui reconnaisse le titre d'héroïne. Ses

41 Dans d'autres études, portant sur divers pays, on note chez les femmes, au XIX siècle, la même tendance à se retirer d'activités politiques qui semblaient normales au siècle précédent. Voir en particulier, Janet L. Polasky, «Women in Revolutionary Belgium: from Stone Throwers to Hearth Tenders», History Workshop, 21 (printemps 1986): 87-104.

42 Dictionnaire biographique du Canada (Québec, Presses de l'Université Laval, 1972), 10: 335; Le Populaire, 12 juillet 1837; Montreal Gazette, 26 octobre 1837.

43 J.-B. Richard, Les Événements de 1837 à Saint-Denis-sur-Richelieu (Saint-Hyacinthe, Société d'histoire régionale de Saint-Hyacinthe, 1938), 21-27; Montreal Gazette, 30 septembre 1837; La Minerve, 12 octobre 1837; The Vindicator, 29 septembre 1837; Le Populaire, 29 septembre, 2 octobre, 9 octobre et 16 octobre 1837; ANC, MG11, Q238-1: 190, Gosford à Glenelg, 12 octobre 1837; Toronto Public Library, le journal de George Nelson, p. 6. 
convictions loyalistes ne viennent sûrement pas de sa famille puisqu'elle est la sœur d'un homme politique patriote et la cousine de Louis-Joseph Papineau. En fait, Rosalie Cherrier semble avoir été complètement coupée du reste de la famille. Quelque temps avant la rébellion, elle a quitté son mari, un maître d'école, et c'est peut-être là l'origine de sa brouille avec sa famille. Comme elle vit à SaintDenis avec ses deux fillettes et un jeune Américain, qui loge chez elle à titre d'invité plus ou moins permanent, elle est une cible de choix pour les ragots sexistes du village. Celle que l'on surnomme «la Poule» est accusée d'être la maîtresse entretenue de Sabrevois de Bleury, un ancien patriote qui s'est rallié au gouvernement, et de garder un jeune homme auprès d'elle pour satisfaire ses appétits dépravés. Et pire encore; la presse radicale rapporte, après ses démêlés avec les patriotes locaux, qu'elle se promène le soir dans les rues de Saint-Denis, bras dessus bras dessous avec son amant, vêtue en sœur de la Congrégation! Déviante à la fois dans sa vie domestique et dans son allégeance politique, Rosalie Cherrier est une femme doublement en désaccord avec sa famille et son milieu.

Forte tête, de toute évidence, Rosalie ne semble pas craindre les affrontements. Elle ne se cache pas pour rédiger des chroniques sur les affaires régionales dans le journal constitutionnel Le Populaire. En septembre 1837, les patriotes de Saint-Denis organisent une assemblée de protestation au cours de laquelle ils pendent en effigie le gouverneur Gosford et plusieurs de ses partisans du Bas-Canada; Rosalie vient arracher les pancartes attachées aux effigies, en clamant devant les spectateurs médusés ses propres opinions sur les problèmes de l'heure. Ce soir-là, une foule bruyante entoure sa maison, au cœur du village, en chantant des refrains obscènes et en réclamant son départ de la paroisse. Mais le lendemain, au lieu de s'enfuir, Rosalie s'achète un fusil et fait fondre ses cuillères pour fabriquer des balles. Le soir, lorsqu'un groupe encore plus nombreux que la veille s'assemble pour reprendre le charivari, madame Saint-Jacques les attend de pied ferme. La foule se montre de plus en plus bruyante et menaçante quand un coup de feu part d'une fenêtre de la maison. On n'a jamais su si c'est Rosalie qui a tiré ou quelqu'un de sa maisonnée sous ses ordres. Chose certaine, lorsque l'agitation se calme, deux hommes qui participaient au charivari gisent sur le sol (l'un d'eux manque mourir), Rosalie et le reste de la maisonnée se sont enfuis et la foule furieuse a réduit la maison à un tas de décombres ${ }^{44}$.

44 Rosalie Cherrier a été capturée le lendemain, amenée à Montréal et accusée de tentative de meurtre; il semble qu'elle ait été acquittée. Malheureusement, les archives judiciaires de cette période ne sont pas encore classées et accessibles aux chercheurs. 
Plus tard, des journalistes se demanderont s'il s'agissait d'un charivari politique ou d'un charivari traditionnel, s'il était dirigé contre l'épouse anticonformiste, l'adversaire partisane, ou contre une femme qui avait osé s'immiscer dans la sphère publique des débats politiques. Il était sans doute tout cela à la fois. Dans le discours républicain de l'époque, pudeur et vertus domestiques féminines sont la contrepartie nécessaire de la vertu civique masculine; par conséquent, aux yeux des villageois de Saint-Denis comme de la bourgeoisie patriote de Montréal, les emportements politiques «immodestes» de Rosalie Cherrier et sa supposée dépravité sexuelle ne font qu'un. Son allégeance au régime colonial ne fait que confirmer à la fois sa corruption et celle du régime.

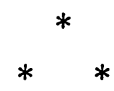

L'échec de la rébellion et la défaite des patriotes n'entraînent évidemment pas d'aucune façon la libération des femmes du BasCanada. La structure gouvernementale et l'ordre politique institués après 1838 sont, au contraire, encore plus entièrement masculins que ne l'a été le régime ébauché par les patriotes ${ }^{45}$. Plus que jamais, on enseigne aux femmes à s'occuper avant tout de leur vie privée, de leur famille. (En pratique pourtant, l'idéologie domestique n'empêche pas les femmes de jouer un rôle important hors du foyer, surtout dans les œuvres de charité, l'éducation et les réformes morales et religieuses; dans le Bas-Canada, l'Église et la vie religieuse offrent des débouchés importants aux filles ambitieuses ${ }^{46}$ ). Mais en dépit de ces initiatives, le régime victorien des «sphères séparées» (pour employer une expression abrégée assez inadéquate) gagne rapidement du terrain ${ }^{47}$.

Dans les années 1830 et 1840 , d'importants changements sont en cours, des changements qui demandent une restructuration en profondeur des relations politiques entre les sexes. L'État acquiert des

45 Voir Lykke de la Cour, Cecilia Morgan et Mariana Valverde, «Gender and State Formation in Nineteenth-Century Canada», Allan Greer et Ian Radforth, eds., Colonial Leviathan: State Formation in Nineteenth-Century Canada (à paraitre).

46 Voir Marta Danylewycz, Profession religieuse. Un choix pour les Québécoises, 18401920 (Montréal, Boréal, 1988).

47 Comme toujours, les distinctions entre public et privé, politique et social, masculin et féminin étaient contestées, changeantes et ambiguës. Il ne s'agit donc pas d'un simple processus par lequel les hommes s'emparent de tout ce qui est "public», tandis que les femmes sont reléguées à une sphère strictement "privée». Pour une critique de cette conception très statique et très mécanique des "sphères distinctes», voir Linda K. Kerber, "Separate Spheres, Female Worlds, Woman's Place: the Rhetoric of Women's History», Journal of American History, 75 (juin 1988): 9-39. 
pouvoirs sans précédent, le contenu de la politique officielle s'appauvrit et les femmes sont de plus en plus coupées des affaires publiques. C'est là un phénomène international que l'on peut observer, sous une forme ou l'autre, un peu partout en Europe occidentale et en Amérique $^{48}$. Associé étroitement à la montée de la bourgeoisie, il n'est pas lié à tel ou tel parti ou faction spécifique ni à un événement politique ou à une révolution en particulier. On peut néanmoins considérer le parti patriote comme le fer de lance, dans le Bas-Canada, de cette mutation générale de l'ordre politico-sexuel. Laissant de côté leurs accès de misogynie intermittante de 1837 , ce sont les patriotes qui ont énoncé le concept démocratique d'une «sphère publique» accessible à tous les citoyens, sans privilège ni distinction. Comme leur définition de la citoyenneté exclut les femmes, leur discours sur la libération a une double portée: sexuelle et politique. Par là même, on serait justifié de considérer la rébellion de 1837-1838, comme, entre autres choses, une étape importante dans la construction des rapports entre le masculin et le féminin au Canada français.

Traduction: Suzanne Mineau

48 Au sujet des États-Unis, voir l'essai révélateur de Paula Baker, «The Domestication of Politics: Women and American Political Society, 1780-1920", American Historical Review, 89 (juin 1984): 620-647. 